\title{
Efeito de diferentes agentes desinfetantes na estabilidade dimensional de materiais de moldagem
}

\author{
Effect of different disinfectant agents on the dimensional stability of molding materials \\ Efecto de diferentes agentes desinfectantes en estabilidad dimensional de materiales de \\ moldeado
}

Gislyane Lima de Queiroz ${ }^{1 *}$, Matheus Duarte de Araújo ${ }^{1}$, Nathaly de Oliveira Queiroz ${ }^{1}$, Talita Arrais Daniel Mendes ${ }^{1,3}$, Luiz Filipe Barbosa Martins ${ }^{1}$, Sofia Vasconcelos Carneiro ${ }^{1}$, Cosmo Helder Ferreira da Silva ${ }^{1}$, Ana Carolina Luna de Carvalho ${ }^{1}$, Victor Pinheiro Feitosa ${ }^{2}$, Mariana Vasconcelos Guimarães ${ }^{1,3}$, Larice Kércia Braz Monteiro ${ }^{1}$, Natasha Muniz Fontes ${ }^{1}$, Jacqueline de Santiago Nojosa ${ }^{1}$, Maria Clara Ayres Estellita ${ }^{3}$, Érika Matias Pinto Dinelly ${ }^{1}$.

\section{RESUMO}

Objetivo: Avaliou-se o efeito de agentes desinfetantes na estabilidade dimensional de materiais de moldagem. Métodos: Por meio de um troquel mestre metálico e uma moldeira compatível foram realizadas 90 moldagens no total, confeccionadas com alginato [AG] $(n=45)$ e silicona de adição [SA] $(n=45)$. Após lavagem em água corrente e secagem os moldes foram subdivididos em três grupos $(n=15)$ de acordo com os métodos de desinfecção, água destilada [AD], controle, clorexidina [CLX] e hipoclorito de sódio [HS]. Realizou-se a lavagem dos moldes em água corrente e os mesmos foram submetidos a jatos de ar. Após desinfecção dos moldes os espécimes pertencentes a CLX e HS foram lavados em água corrente e receberam jatos de ar. Os corpos de prova tiveram seus diâmetros mensurados por meio de um paquímetro digital em dois momentos distintos. Realizou-se o teste ANOVA - one way, Tukey e teste $t(P<0,05)$. Resultados: Os espécimes demonstraram alteração significativa após cinco dias de armazenamento, sendo que AD e grupo hipoclorito de sódio não obtiveram alterações. Conclusão: O método mais indicado para desinfecção do AG foi a CLX, que em contrapartida mostra-se prejudicial ao SA, sendo indicado o HS.

Palavras-chave: Materiais para Moldagem Odontológica, Desinfecção, Hipoclorito de Sódio, Clorexidina.

\begin{abstract}
Objective: The effect of disinfectants on the dimensional stability of molding materials was evaluated. Methods: A total of 90 moldings were made using alginate $[A G](n=45)$ and addition silicone $(S A)(n=45)$ using a metal master die and a compatible tray. After washing in running water and drying the molds were subdivided into three groups $(n=15)$ according to disinfection methods, distilled water [AD], control, chlorhexidine $[\mathrm{CLX}]$ and sodium hypochlorite $[\mathrm{HS}]$. The molds were rinsed under running water and they were subjected to air jets. After disinfection of the molds the specimens belonging to CLX and HS were washed in running water and received air jets. The specimens had their diameters measured by means of a digital caliper at two different moments. ANOVA - one way, Tukey and $t$ test $(P<0.05)$ were performed. Results: The specimens showed significant alteration after five days of storage, and $A D$ and sodium hypochlorite group did not change. Conclusion: The most suitable method for disinfecting the GA was the CLX, which in turn is harmful to the SA, being indicated for this HS.
\end{abstract}

Keyworlds: Dental Impression Materials, Disinfection, Sodium Hypochlorite, Chlorhexidine.

${ }^{1}$ Centro Universitário Católica de Quixadá. Quixadá-CE. *E-mail: gislyane.lqc@gmail.com

2 Faculdade Paulo Picanço. Fortaleza-CE

3Universidade Federal do Ceará. Fortaleza-CE

SUBMETIDO EM: 5/2019

ACEITO EM: 6/2019

PUBLICADO EM: 7/2019

REAS/EJCH | Vol. Sup.27 | e916 | DOI: https://doi.org/10.25248/reas.e916.2019 Página 1 de $\mathbf{6}$ 


\section{RESUMEN}

Objetivo: Se evaluó el efecto de agentes desinfectantes en la estabilidad dimensional de los materiales de moldeo. Métodos: Por medio de un troquel maestro metálico y una cubeta compatible se realizaron 90 moldes en total, confeccionados con alginato $[A G]$ y silicona de adición [SA] $(n=45)$. Después de lavado en agua corriente y secado, los moldes fueron subdivididos en tres grupos de acuerdo con los métodos de desinfección, agua destilada [AD], control, clorexidina [CLX] e hipoclorito de sodio [HS]. Se realizó el lavado de los moldes en agua corriente y los mismos fueron sometidos a chorros de aire. Después de desinfección de los moldes, los especímenes pertenecientes a CLX y HS fueron lavados en agua corriente y recibieron chorros de aire. Los cuerpos de prueba tuvieron sus diámetros medidos por medio de un paquímetro digital en dos momentos distintos. Se realizó la prueba ANOVA - un way, Tukey $(P<0,05)$. Resultados: Los especímenes mostraron un cambio significativo después de cinco días de almacenamiento, siendo que AD y hipoclorito de sodio no obtuvieron alteraciones. Conclusión: El método más indicado para desinfección del AG fue la CLX, que en contrapartida se muestra perjudicial al SA, siendo indicado para este el HS.

Palabras clave: Materiales de Impresión Dental, Desinfección, Hipoclorito de Sodio, Clorhexidina.

\section{INTRODUÇÃO}

As impressões feitas com materiais de moldagem são consideradas a fonte principal de contaminação cruzada entre dentistas e pacientes. No procedimento de moldagem, eles entram em contatos com fluidos bucais, como saliva, sangue, exsudatos e outros, que podem conter microrganismos patogênicos, possibilitando a transmissão de doenças infectocontagiosas (BOER PR, FRANCISCONI PAS, FROSSARD $M, 2004)$.

Considerando a importância da biossegurança, tanto para a equipe profissional, quanto para os pacientes, visto que a esterilização é um método danoso aos materiais de moldagem, procedimentos como a desinfecção por imersão, aonde soluções químicas vêm sendo utilizadas, tem sido considerado um método bastante eficaz. Com isso, os profissionais têm que ter cautela quanto a contaminação cruzada, reduzindo as taxas de transmissão de infecção de laboratórios dentários. A International Dental Federation (IDF) persiste em fazer a antissepsia de todas as impressões tiradas de pacientes antes do envio para os laboratórios (BOER PR, FRANCISCONI PAS, FROSSARD M, 2004).

Atualmente no mercado, há uma grande variedade de antimicrobianos aplicados para a desinfecção dos moldes odontológicos. No entanto, devem ser levados em consideração alguns fatores, como garantir que o produto seja eficaz e que a composição química desses não altere a estabilidade dimensional e a capacidade de reprodução de detalhes do material de moldagem, dentre os quais destacam-se o Alginato e a Silicona de Adição (RENTZIA A, 2011).

O alginato é indicado para a obtenção de modelos de estudo, registro intermaxilar, confecção de prótese provisória, moldeiras especializadas, próteses maxilofaciais, prótese total, entre outros (ANDRADE IM, et al., 2014). Quanto a sua seleção como material de moldagem, faz-se necessário que o profissional conheça os diferentes produtos disponíveis no mercado e compare suas diferentes marcas, a fim de escolher um material que não apresente grandes alterações volumétricas (BRAGA AS, et al., 2012).

Os alginatos convencionais possuem uma instabilidade dimensional fazendo com que fossem desenvolvidos alginatos de última geração com o objetivo de melhorar essa desvantagem, visto que esse tipo de material é bastante utilizado nas práticas odontológicas. Conforme preconizam os fabricantes, os alginatos de última geração apresentam uma melhor estabilidade dimensional, aumentando o seu tempo de vazamento por até cinco dias, quando armazenados corretamente em recipiente plástico hermeticamente selado (IMBERY TA, et al., 2010). O silicone de adição é um material de moldagem bastante solicitado nas práticas de moldagem devido a sua excelente capacidade de reprodução de detalhes anatômicos, propriedades físicas e elásticas, estabilidade dimensional, permanecendo até 7 dias por não liberar subprodutos, permite mais de um vazamento do mesmo molde, além de ser bem aceito pelo paciente por apresentar um sabor agradável. Apesar de ter alto custo, é um elastômero que tem o seu uso considerável em moldagens de pacientes 
desdentados totais ou parciais, quando o caso requer uma melhor impressão de detalhes (HAMALIAN TA, NASR E, CHIDIAC JJ, 2012). Apresenta melhor estabilidade dimensional em relação aos outros materiais de moldagem $(0,05 \%)$, seguido do poliéter $(0,15 \%)$, silicone de condensação $(0,50 \%)$ e polissulfeto $(0.60 \%)$. São indicados para realização de moldagens unitárias, moldagens por quadrante e moldagens totais para dentados, desdentados parcial ou total (MESQUITA VT, et al., 2012).

Fonseca RG et al. (2007) relatam que os moldes devem ser lavados em água corrente para eliminar detritos, saliva ou sangue, e posteriormente imergi-los em soluções químicas compatíveis com as propriedades dos materiais de moldagem, seguindo as normas de biossegurança preconizadas pela American Dental Association (ADA) (FONSECA RG, et al., 2007).

Uma vez que a variedade de produtos para desinfecção de moldes odontológicos é extensa, o presente estudo buscou avaliar a interferência na estabilidade dimensional de dois antimicrobianos amplamente utilizados pelos profissionais, representados pelo Digluconato de Clorexidina 2\% e Hipoclorito de Sódio 1\%, sobre moldes de alginato de última geração e silicona de adição, desinfetados e mensurados em um primeiro momento imediatamente após a moldagem e posteriormente mensurados após cinco dias da moldagem. A hipótese do presente estudo se dá que os agentes de desinfecção não irão influenciar na estabilidade dimensional de diferentes materiais de moldagem.

\section{MÉTODOS}

O presente trabalho trata-se de um estudo laboratorial in vitro, no qual foi utilizada como corpo de prova materiais de moldagens disponíveis para compra, sem necessidade de aprovação do comitê de ética de seres humanos. Esta pesquisa buscou o conhecimento do melhor produto para desinfecção do alginato [AG] de última geração Hydrogum $5 \circledast$ (Zhermack/Badia - Polesine, Itália) bem como da silicona de adição [SA] Scan Putty® (Yller- Pelotas, Rio grande do Sul - Brasil) frente a alteração de sua estabilidade dimensional no período imediato e com cinco dias após a realização do procedimento de moldagem. Por meio de um troquel mestre metálico e uma moldeira compatível foram realizadas 90 moldagens confeccionadas com alginato ( $\mathrm{n}$ $=45)$ e silicona de adição $(n=45)$. Após lavagem em água corrente por 1 minuto e secagem com jatos de ar por 5 segundos através da seringa triplice, os moldes foram subdivididos em três grupos $(n=15)$ de acordo com os métodos de desinfecção: AD - moldes sem desinfecção (controle) lavados com água destilada ; HS moldes desinfetados com hipoclorito de sódio a 1\% e CLX - moldes desinfetados com digluconato de clorexidina a $2 \%$ [CLX] (Tabela 1).

Tabela 1 - Materiais utilizados na desinfecção e forma de desinfecção.

\begin{tabular}{lllll}
\hline PRODUTO & Fabricante & Composição & Utilização & Tempo de ação \\
\hline CLOREXIDINA 2\% & Vilevie ${ }^{\circledR}$ & $\begin{array}{l}\text { Digluconato de } \\
\text { clorexidina a 2\% }\end{array}$ & Imersão do molde & 10 minutos \\
DESINFECTANTE & Rioquímica ${ }^{\circledR}$ & $\begin{array}{l}\text { Hipoclorito de } \\
\text { sódio 1\% }\end{array}$ & Imersão do mole & 10 minutos \\
CLORO RIO 1\% & & & \\
\hline
\end{tabular}

${ }^{*}$ Os moldes desinfetados foram acondicionados em uma caixa plástica hermeticamente fechada durante 0 tempo indicado para ação do agente químico.

Fonte: Dados da Pesquisa 2019.

Para obtenção dos espécimes, o procedimento padrão de desinfecção foi realizado da mesma forma alterando apenas o material de moldagem. Em seguida, realizou-se a lavagem dos moldes por 1 minuto em água corrente e os mesmos foram submetidos a leves jatos de ar através da seringa tríplice por 5 segundos. Após desinfecção dos moldes por 10 minutos com o método de imersão, os corpos de prova pertencentes aos grupos 2 e 3 foram novamente lavados em água corrente por 1 minuto e, em seguida, receberam leves jatos de ar com seringa tríplice por 5 segundos. 
Após a obtenção e desinfecção dos corpos de provas os mesmos tiveram suas dimensões mensuradas através de um paquímetro digital (Digimess Instrumentos de Precisão Ltda - São Paulo, Brasil), para verificar a mensuração imediata. Os corpos de prova foram armazenados em uma caixa hermeticamente selada no período de cinco dias, período para a realização da segunda mensuração, resultando em um total de 180 mensurações. A partir dos dados obtidos foram realizadas análises estatísticas utilizando o teste Shapiro-Wilk para avaliação da normalidade dos dados, seguido de uma análise de variância ANOVA - one way e Tukey e teste $t(p<0,05)$.

\section{RESULTADOS}

Pode-se observar que no material AG, em um período imediato, o HS apresentou diferença estatisticamente significativa $(6,4 \pm 0,2 \mathrm{~mm})$ dos demais meios de desinfecção. Quando comparou-se diferentes tempos nesse mesmo material de moldagem observou-se que a deformação do material se mostrou maior com 5 dias de avaliação em todos os grupos. Após 5 dias de armazenamento observou-se que $A D$ e CLX foram semelhantes, sendo que o HS foi a solução desinfetante com pior desempenho quanto a estabilidade dimensional, nesse tempo $(p<0,05)$, descrito na Tabela 2.

Quando analisamos o material de moldagem SA sob diferentes formas de desinfecção observamos que imediatamente o grupo CLX apresentou uma maior estabilidade dimensional quando comparado aos demais. Já em uma avaliação após 5 dias não obteve-se diferença estatisticamente significativa, sendo todos os grupos apresentaram semelhança na estabilidade dimensional. Se avaliarmos cada meio de desinfecção em diferentes tempos apenas a CLX obteve diferença estatística, sendo sua maior deformação após 5 dias (6,4 $\pm 0,2$ ) (Tabela 2).

Tabela 2 - Médias e desvios padrões da análise de alteração dimensional $(\mathrm{mm})$ considerando o tipo de material de moldagem, material de desinfecção e tempo de armazenamento.

\begin{tabular}{cccc}
\hline & \multicolumn{3}{c}{ Material de Desinfecção x Tempo } \\
\hline \multirow{2}{*}{ Materiais de Moldagem } & $\mathrm{AD}$ & $\mathrm{CLX}$ & $\mathrm{HS}$ \\
\cline { 2 - 4 } & IMEDIATO & IMEDIATO & IMEDIATO \\
\hline \multirow{2}{*}{$\mathrm{AG}$} & $6,3 \pm 0,1 \mathrm{ABb}$ & $6,3 \pm 0,2 \mathrm{Bb}$ & $6,4 \pm 0,2 \mathrm{Ab}$ \\
& $5 \mathrm{DIAS}$ & $5 \mathrm{DIAS}$ & $5 \mathrm{DIAS}$ \\
& $6,5 \pm 0,2 \mathrm{Ba}$ & $6,4 \pm 0,1 \mathrm{Ba}$ & $6,6 \pm 0,1 \mathrm{Aa}$ \\
\cline { 2 - 4 } & IMEDIATO & IMEDIATO & IMEDIATO \\
\cline { 2 - 4 } $\mathrm{SA}$ & $6,5 \pm 0,1 \mathrm{Aa}$ & $6,21 \pm 0,16 \mathrm{Bb}$ & $6,4 \pm 0,1 \mathrm{Aa}$ \\
& $5 \mathrm{DIAS}$ & $5 \mathrm{DIAS}$ & $5 \mathrm{DIAS}$ \\
& $6,4 \pm 0,1 \mathrm{Aa}$ & $6,4 \pm 0,1 \mathrm{Aa}$ & $6,5 \pm 0,1 \mathrm{Aa}$ \\
\hline
\end{tabular}

Legenda: Letras maiúsculas representam diferenças estatisticamente significativas entre linhas (material de desinfecção) $(p<0,05)$. Letras minúsculas representam diferenças estatisticamente significativas entre 0 tempo (imediato $\times 5$ dias) $(p<0,05)$.

Fonte: Dados da Pesquisa 2019.

\section{DISCUSSÃO}

Um problema bastante vigente nos dias atuais é a infecção cruzada ente o consultório odontológico e os laboratórios protéticos, pois a descontaminação não é uma realidade, isso pode -se dar ao fato de que os meios de desinfecção podem gerar uma deformação dos moldes em questão comprometendo assim a fidedignidade dos modelos, para posterior confecção dos trabalhos. Assim sendo, a hipótese do estudo foi rejeitada, sendo que os meios de desinfecção influenciaram na estabilidade dimensional dos modelos. 
Lemos IS et al. (2010), analisaram algumas características do hipoclorito de sódio a 1\%, que possui função intermediária de largo espectro de combate a vírus e bactérias, além de possuir baixo custo. Possui eficiente ação antimicrobiana, com ação comprovada em 10 minutos. Essa concentração permite que ele possa agir sobre o vírus da hepatite $B$, Mycobacterium tuberculosis (bactéria causadora da tuberculose), entre outros.

No presente estudo, o tempo de desinfecção para os moldes de Alginato e Silicona de Adição foi estabelecido como 10 minutos (Tabela 1), visto que, de acordo com Yilmaz H et al. (2007), esse tempo pode ser preconizado para que espécimes de poliéster sejam descontaminados dos fluidos bucais sem gerar alterações significativas na sua estabilidade dimensional quando comparado ao grupo controle, que não manteve contato com soluções desinfetantes. Foi preconizado o tempo de 10 minutos para desinfecção dos corpos de prova para avaliar se o hipoclorito de sódio a $1 \%$ e a clorexidina a $2 \%$ provocariam modificações significativas na estabilidade dimensional de moldes de alginato e silicone de adição durante esse tempo (LEMOS IS, et al., 2010).

Ao avaliar a estabilidade dimensional do alginato comprovaram que o alginato reage à imersão em solução desinfetante num tempo determinado por possuir composições que restringem um contato excessivo com materiais de desinfecção. A presente pesquisa buscou avaliar se este método de desinfecção possui eficácia, embora existam outras técnicas que também mostraram mais eficácia quanto a preservação da estabilidade dimensional e do controle microbiológico, como por exemplo, spray e nebulização de hipoclorito de sódio (MOURA CDVS, et al., 2010).

Alginatos de última geração como Carvex ColorChange $\AA^{\circledR}$ e Hydrogum $5 \AA$ apresentam suas dimensões favoráveis quando são armazenados seguindo um padrão de tempo e local adequados para que haja a obtenção de modelos de estudo e, possivelmente, de estruturas para próteses parciais, entre outros. $O$ alginato utilizado nesta pesquisa foi o Hydrogum 5, pois ele estatisticamente, apresenta menos interferências em sua estabilidade dimensional durante 5 dias, quando é comparado ao Cavex Color Change (FERNANDES SL, et al., 2014).

Iwasaki $\mathrm{Y}$ et al. (2016), avaliaram a estabilidade dimensional de moldes que foram submetidos a desinfecção com hipoclorito de sódio a 0,5\% entre grupos nos tempos 1, 3, 5, 10 minutos e concluíram que os valores apresentados pelo grupo controle foram inferiores quando comparados aos que utilizaram a solução de hipoclorito de sódio a $0,5 \%$, entretanto, a solução por até 10 minutos não gerou prejuízos significativos às propriedades do material. Divergindo aos resultados da presente pesquisa, que faz uso da solução de hipoclorito de sódio em uma concentração maior (1\%), pois, após a etapa de desinfecção de moldes de alginato é possível observar uma diferença significativa após 5 dias de armazenamento dos espécimes (IWASAKI Y, et al., 2016).

Ao avaliarem o tempo de desinfecção ideal para que não alterasse os moldes, Gounder R e Vikas BV (2016), observaram que a Silicona de Adição, após 60 minutos em contato com a solução de hipoclorito a $1 \%$, apresentou pequenas alterações em sua dimensão. Os resultados obtidos mostraram a variação de $0,024 \%$ a $0,05 \%$. Já na presente pesquisa utilizou-se a desinfecção com o hipoclorito a $1 \%$ em menos tempo (10 minutos) e obteve-se um valor de $p>0,05$ quando comparados com o grupo controle.

Amalan A et al. (2013), obtiveram resultados significativos comparando a interação dos desinfetantes com os hidrocolóides irreversíveis disponíveis comercialmente. Observou-se que o Digluconato de Clorexidina aumentou o tempo de geleificação do alginato, todavia, diminuiu a resistência do gel. Já o Hipoclorito de Sódio reduziu o tempo de presa do alginato e a reprodução de detalhes do material, mas também, aumentou a resistência do gel. De acordo com os dados, a Clorexidina pode ser o material de escolha para desinfecção de hidrocolóides, pois não gera alterações significativas. O que corrobora com a presente pesquisa, que utiliza a Clorexidina numa concentração a 2\% (AMALAN A, et al., 2013).

Para este estudo preconizou-se um período padrão para realizar as mensurações dos espécimes. As primeiras medições foram obtidas imediatamente após moldagem (Grupo Controle) e desinfecção (Grupos Experimentais) e, após 5 dias de armazenamento dos moldes em uma caixa hermeticamente fechada, uma vez que, de acordo com o fabricante do Hydrogum 5 , ele pode ser vazado até 5 dias. E ainda, a Silicona de 
Adição utilizada foi somente do tipo pesada, sendo que os pesquisadores não aguardaram o período de 1 hora (tempo para vazar o gesso) para realizar as mensurações por questões de padronização entra a avaliação dos produtos (Tabela 2). Dessa forma, obteve-se resultados significativos e de bastante relevância para a literatura.

\section{CONCLUSÃO}

Apesar da inconveniente alteração na estabilidade dimensional dos moldes frente aos métodos desinfetantes, faz-se necessário a desinfecção destes independentemente do material de moldagem usado, pois a negligência nesta etapa clínica pode ocasionar contaminações diversas. Sendo assim, pode-se concluir que no AG o tempo de armazenamento influencia na deformação do material de moldagem, bem como o HS não é o material mais indicado para essa função de desinfecção, pois causa uma maior alteração dimensional. Já na SA, deve-se ressaltar que o material mais indicado para isso é a CLX, numa imersão imediata, no entanto, com o passar dos dias, essa desinfecção pode deformar o molde, independente do material utilizado para isso.

\section{AGRADECIMENTOS}

Ao programa de Pós-graduação em Odontologia da Universidade Federal do Ceará.

\section{REFERÊNCIAS}

1. ANDRADE IM, et al. Trial of an experimental castor oil solution for cleaning dentures. Brazilian Dental Journal, 2014; $25(1)$.

2. Braga AS, et al. Potencial tóxico dos alginatos para uso odontológico. Revista de Ciências Farmacêuticas Básica e Aplicada, 2007; 28 (2).

3. IMBERY TA, et al. Accuracy and dimensional stability of extended-pour and conventional alginate impression materials. The Journal of the American Dental Association. 2010; 141(1).

4. HAMALIAN TA, et al. Impression Materials in Fixed Prosthodontics: Influence of Choice on Clinical Procedure. Journal of Prosthodontics, 2011; 20 (2).

5. MESQUITA VT, et al. Materiais e técnicas de moldagem em prótese fixa- revisão de literatura. Saber científico odontológico, 2012; 2 (1).

6. BOER PR, et al. Avaliação dimensional de troquéis de gesso obtidos de moldes de hidrocolóide irreversível após desinfecção. Ciências Biológicas e da Saúde, 2004; 25 (1).

7. RENTZIA A. Disinfection procedures: their efficacy and effect on dimensional accuracy and surface quality of an irreversible hydrocolloid impression material. Journal of Dentistry, 2011; 39 (2).

8. FONSECA RG, et al. A influência da imersão em ácido peracético sobre a reprodução de detalhes e compatibilidade dos elastômeros com gesso. Revista Odonto Ciência, 2007; 22.

9. LEMOS IS, et al. Avaliação da alteração dimensional de modelos obtidos a partir de moldagens com alginato tradicional e outro com desinfetante. Revista de Odontologia da UNESP, 2010; 39 (1).

10. YILMAZ $\mathrm{H}$, et al. Effect of disinfection on the dimensional stability of polyether impression materials. Journal of Prosthodontics, 2007; 16 (6).

11. MOURA CDVS, et al. Disinfection of irreversible hydrocolloid impressions with sodium hypochlorite steam: assessment antimicrobial efficacy. Revista Odonto Ciência, 2010; 25 (2).

12. FERNANDES SL, et al. Estabilidade dimensional linear de alginatos de última geração em função do tempo de armazenagem dos moldes. Innovations Implant Journal: Biomaterials and Esthetics, 2014.

13. IWASAKI Y, et al. Effects of immersion disinfection of agar-alginate combined impressions on the surface properties of stone casts. Dental Materials Journal, 2016; 35(1).

14. GOUNDER R, VIKAS BV. Comparison of disinfectants by immersion and spray atomization techniques on the linear dimensional stability of different interocclusal recording materials: An in vitro study. European Journal of Dentistry. 2016; 10 (1).

15. AMALAN A, et al. Evaluation of properties of irreversible hydrocolloid impression materials mixed with disinfectant liquids. Dental Research Journal, 2013; 10 (1). 